\title{
Traumatismo abdominal contuso manejado con embolización mesentérica: reporte de caso
}

\author{
Blunt abdominal trauma managed with mesenteric embolization: case report
}

\author{
Álvaro E. Sánchez-Hernández ${ }^{*}$, Iván Martín-González², Rubén Luna-Álvarez², Brandon Steven-Aparicio³, \\ Luis F. Cabrera ${ }^{1}$, Steven Loaiza ${ }^{3}$ y Stefanie Marmolejo ${ }^{3}$ \\ ${ }^{1}$ Departamento de Cirugía General, Universidad El Bosque; ${ }^{2}$ Departamento de Cirugía General, Fundación Clínica Abood Shaio; ${ }^{3}$ Departamento \\ de Medicina, Universidad El Bosque. Bogotá, Colombia
}

\begin{abstract}
Resumen
El trauma abdominal cerrado se presenta en el $20 \%$ de los pacientes politraumatizados, de los cuales aproximadamente el $5 \%$ pueden llegar a tener lesiones mesentéricas. Presentamos el caso de una mujer que sufre un traumatismo abdominal cerrado a la cual se le realizó tomografía computarizada con evidencia de un hematoma en el flanco derecho, que se logra controlar por angioembolización de la arteria mesentérica superior. El tratamiento de las lesiones mesentéricas se determinará según el estado hemodinámico del paciente, pues ante la estabilidad se puede escoger entre cirugía abierta y embolización, pero en caso de inestabilidad se recomienda la laparotomía.
\end{abstract}

Palabras clave: Lesión mesentérica. Tratamiento. Embolización. Laparotomía.

\begin{abstract}
Blunt abdominal trauma occurs in $20 \%$ of polytraumatized patients, of which approximately $5 \%$ may have mesenteric lesions. We present a case of a woman suffering from a blunt abdominal trauma, who underwent computed tomography with evidence of hematoma on the right flank, which was controlled by angioembolization of the superior mesenteric artery. Treatment of mesenteric lesions will be determined according to the hemodynamic status of the patient, since in view of stability, a choice can be made between open surgery and embolization, but in case of instability, laparotomy is recommended.
\end{abstract}

Key words: Mesenteric injury. Treatment. Embolization. Laparotomy.

\section{Correspondencia:}

*Álvaro E. Sánchez-Hernández

Calle 141 \# 7 b - 37 Apartamento 703

Edificio Michelle

Fecha de recepción: 11-07-2020

C.P. 110111, Bogotá, Colombia

E-mail: appaloosa1991@gmail.com
Fecha de aceptación: 14-10-2020

DOI: $10.24875 / C I R U .20000750$
Cir Cir. 2021;89(6):822-826

Contents available at PubMed www.cirugiaycirujanos.com 0009-7411/@ 2020 Academia Mexicana de Cirugía. Publicado por Permanyer. Este es un artículo open access bajo la licencia CC BY-NC-ND (http://creativecommons.org/licenses/by-nc-nd/4.0/). 


\section{Introducción}

El trauma abdominal cerrado se presenta en el $20 \%$ de los pacientes politraumatizados ${ }^{1}$. Este tipo de lesiones tienen una alta mortalidad cuando el diagnóstico y el tratamiento se retrasan ${ }^{2}$, ya que pueden comprometer varios órganos, como el bazo (40-55\%) y el hígado (35-45\%), entre otros ${ }^{3,4}$.

La lesión mesentérica es una complicación poco frecuente, que se presenta en menos del $5 \%$ de los pacientes con lesiones abdominales directas ${ }^{1,2,5,6 ;}$ sin embargo, ha ido en aumento en los últimos tiempos por los accidentes de automóviles en relación con el cinturón de seguridad ${ }^{1,2}$.

El tratamiento de elección es la laparotomía ${ }^{2}$, pero en los últimos años se ha visto que la embolización es una opción segura ${ }^{1,2}$. Presentamos el caso de una paciente manejada con embolización mesentérica posterior a un traumatismo abdominal cerrado en el flanco y la fosa iliaca derechos, y una revisión de la literatura.

\section{Caso clínico}

Mujer de 20 años que fue trasladada al servicio de urgencias con un cuadro de 1 hora de evolución por traumatismo abdominal en el flanco y la fosa iliaca derechos, al ser atropellada por una bicicleta. Al ingreso, los signos vitales eran: presión arterial 106/75 mmHg, frecuencia cardiaca 66 latidos por minuto, frecuencia respiratoria 14 respiraciones por minuto y saturación de oxígeno del $96 \%$. En la exploración física muestra dolor de gran intensidad a la palpación en el hemiabdomen derecho, sin signos de irritación peritoneal y sin estigmas de traumatismo penetrante.

Dentro de los paraclínicos de ingreso, la hemoglobina fue de $12.2 \mathrm{~g} / \mathrm{dl}$. Se realizó protocolo FAST (Focused Abdominal Sonography for Trauma) y se observó una adecuada contractilidad miocárdica, sin presencia de líquido libre intraperitoneal ni pericárdico. Además, por el traumatismo de alto impacto se indicó la realización de tomografía computarizada abdominal contrastada y traslado a la unidad de cuidado intensivo ( $\mathrm{UCl}$ ) para monitoreo hemodinámico, considerando alto riesgo de inestabilidad hemodinámica, en el contexto de traumatismo abdominal contuso y con posible lesión de víscera sólida.

En el estudio tomográfico se descartaron perforación de víscera hueca y lesión de órgano sólido, y se reportó moderada cantidad de líquido perihepático,

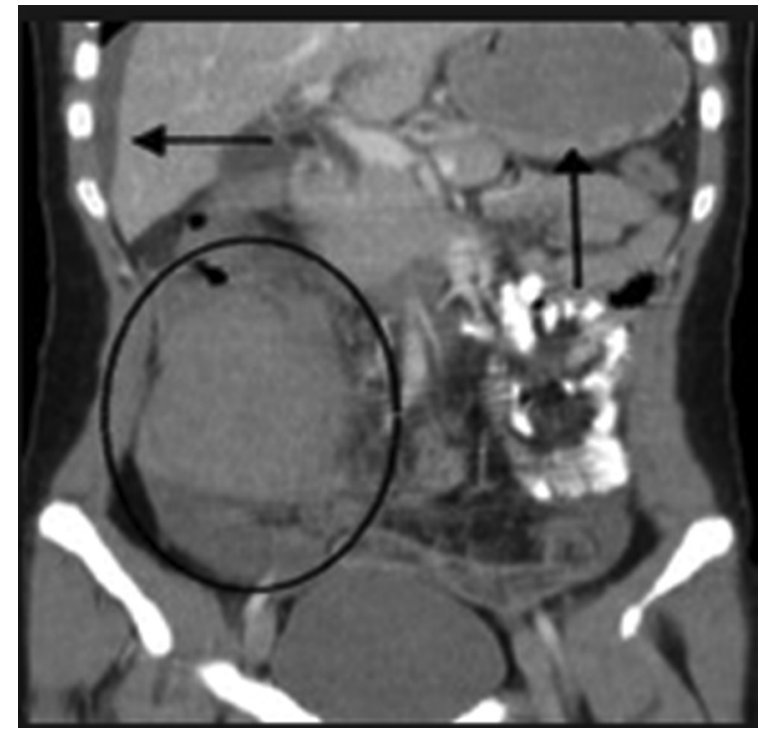

Figura 1. Hematoma intraabdominal en el flanco y la fosa iliaca derechos (círculo), y líquido perihepático (flecha horizontal) y periesplénico (flecha vertical).

periesplénico, en goteras parietocólicas, fosas ilíacas y pelvis menor. En el flanco derecho se identifica una imagen redondeada de contornos regulares, ligeramente hiperdensa, con densidad promedio de $58 \mathrm{UH}$, que mide $42 \times 101 \times 64 \mathrm{~mm}$ y ejerce efecto de masa sobre las asas intestinales, sugiriendo como primera posibilidad un hematoma (Fig. 1).

Durante la estancia en $\mathrm{UCl}$ descendió la hemoglobina progresivamente, con un valor al tercer día de $7.9 \mathrm{~g} / \mathrm{dl}$, por lo cual se indicó una transfusión de tres unidades de glóbulos rojos y valoración por el servicio de radiología intervencionista para realización de embolización arterial por vía percutánea.

El servicio de radiología intervencionista realizó una arteriografía selectiva de la circulación abdominal, específicamente de la arteria mesentérica superior, con un catéter Cobra $5 \mathrm{Fr}$, obteniendo una imagen confirmatoria de un gran hematoma en el flanco y el hipocondrio derecho que ejerce efecto de masa, además de sangrado proveniente de las ramas de la arteria mesentérica superior, por lo que se indicó embolización del vaso sanguíneo que daba lugar al hematoma. Se insertó un microcatéter $2.7 \mathrm{Fr}$ hasta las ramas nutricias descritas, a las cuales se les realizó embolización con micropartículas coils de 100$300 \mu \mathrm{m}$, obteniendo un enlentecimiento del flujo sanguíneo, y posteriormente a través del mismo catéter se avanzaron tres coils (catéter en espiral) y se logró la oclusión del vaso sanguíneo que irrigaba el 
hematoma. Se realizó una arteriografía de control que mostró la adecuada oclusión del flujo del vaso (Fig. 2).

La paciente tuvo una evolución satisfactoria posterior al procedimiento, manteniendo la hemoglobina estable en $10.7 \mathrm{~g} / \mathrm{dl}$, sin episodios de inestabilidad hemodinámica y con mejoría del dolor. Por ello, se indicó el egreso hospitalario al octavo día. En el posterior control ambulatorio no hubo evidencia de complicaciones.

\section{Discusión}

Las lesiones mesentéricas son causadas por diferentes mecanismos: aumento de las presiones, desaceleración, contusión o compresión directa ${ }^{7}$. Se presentan principalmente a nivel de la válvula ileocecal y el músculo suspensor del duodeno ${ }^{2,8}$. Dentro de las principales complicaciones está la alteración de la arteria mesentérica que va a llevar a una hemorragia activa y con el tiempo a desarrollar hematomas compresivos a nivel intestinal, que terminarán en un proceso isquémico ${ }^{2}$.

La mortalidad se eleva cuando no son oportunos el diagnóstico y el tratamiento ${ }^{2,5,9}$, ya que si estos se establecen en menos de 8 horas puede ser del $2 \%$, pero si pasan más de 24 horas puede aumentar hasta el $30.8 \%{ }^{5}$. Idealmente, la institución donde sea atendido el paciente debe tener disponibilidad de cirujano, anestesiólogo, intensivista y radiólogo intervencionista'.

El diagnóstico no se puede basar únicamente en la exploración física, ya que al inicio los lesionados pueden ser asintomáticos o presentar dolor y contracción involuntaria del abdomen. Aunque estos sean signos importantes tras un traumatismo, no son indicaciones de ninguna intervención. Por otro lado, se debe tener en cuenta que después de un trauma puede haber alteraciones del estado de consciencia o lesiones raquimedulares, que afectarían la interpretación semiológica de los signos; además, los pacientes pueden estar bajo los efectos del alcohol o de sustancias psicoactivas. Por todo ello, hay que correlacionar la evaluación física con el estudio de imágenes para un dictamen más específico?.

La evaluación primaria de estos pacientes deben incluir el estado hemodinámico y la identificación de lesiones potencialmente letales, para definir la necesidad de manejo expectante o quirúrgico ${ }^{1,2,8}$. Los que presenten inestabilidad hemodinámica requieren la realización de FAST para establecer la presencia de aire o líquido libre, y si el origen del shock hemorrágico es intraabdominal. Por otro lado, los pacientes

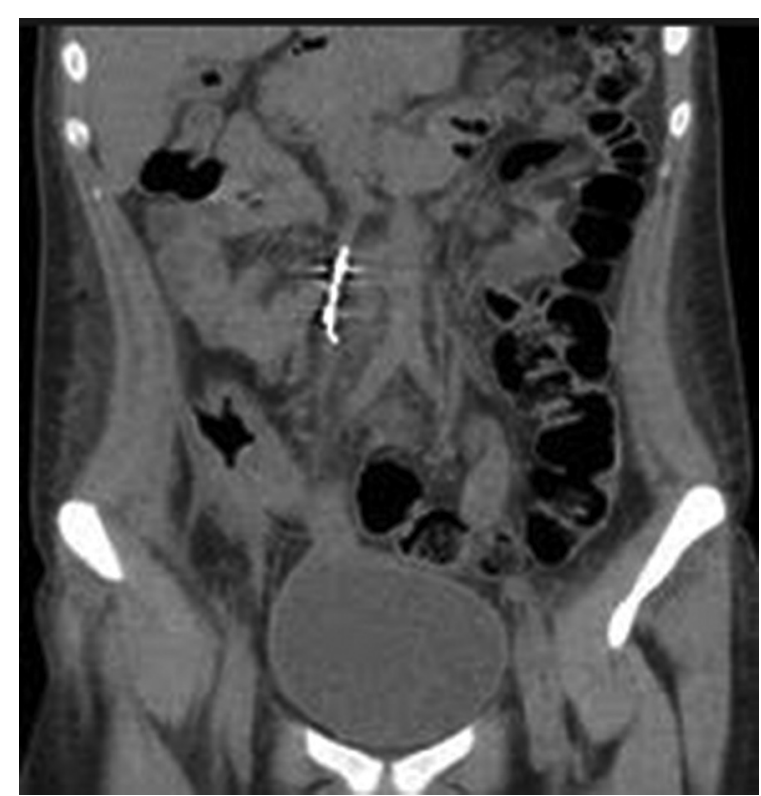

Figura 2. Arteria íleo bicecoapendiculocolica embolizada y disminución del hematoma.

estables y los que responden a manejo con líquidos tienen indicación de tomografía computarizada abdominal contrastada ${ }^{1}$ para determinar el origen del sangrado y descartar lesiones secundarias al trauma ${ }^{1,10}$; además, según lo reportado por Frandon, et al. ${ }^{1}$, el uso de esta prueba diagnóstica ayuda a reducir la mortalidad y los costos hospitalarios. En nuestro hospital realizamos FAST por protocolo a todos los pacientes para entrenamiento, por lo cual a esta paciente estable se le realizó dicha ecografía.

El tratamiento de estas lesiones se puede hacer de dos maneras diferentes: cirugía o embolización ${ }^{2,11}$. La técnica quirúrgica abierta aún sigue siendo de elección ${ }^{2,4}$. Aunque la embolización ya fue descrita en 1974 por Bookstein, cayó en desuso por parte del personal médico ${ }^{12}$ y en la actualidad se vuelve a utilizar porque ha demostrado ser fiable y efectiva ${ }^{1,2}$, como se vio en nuestra paciente.

Los pacientes hemodinámicamente inestables, que no respondan al manejo inicial y que en la ecografía muestren líquido libre en la cavidad peritoneal, deben ser llevados a laparotomía de emergencia para control de la hemorragia, revisión de la cavidad abdominal y descompresión del hematoma si se ha formado ${ }^{1,2}$. Sin embargo, se ha visto que en estos pacientes también se puede hacer una embolización si la cirugía abierta se retrasa, ya que permite realizar un control inicial del sangrado y se facilita la intervención quirúrgica. Haciendo un abordaje combinado 


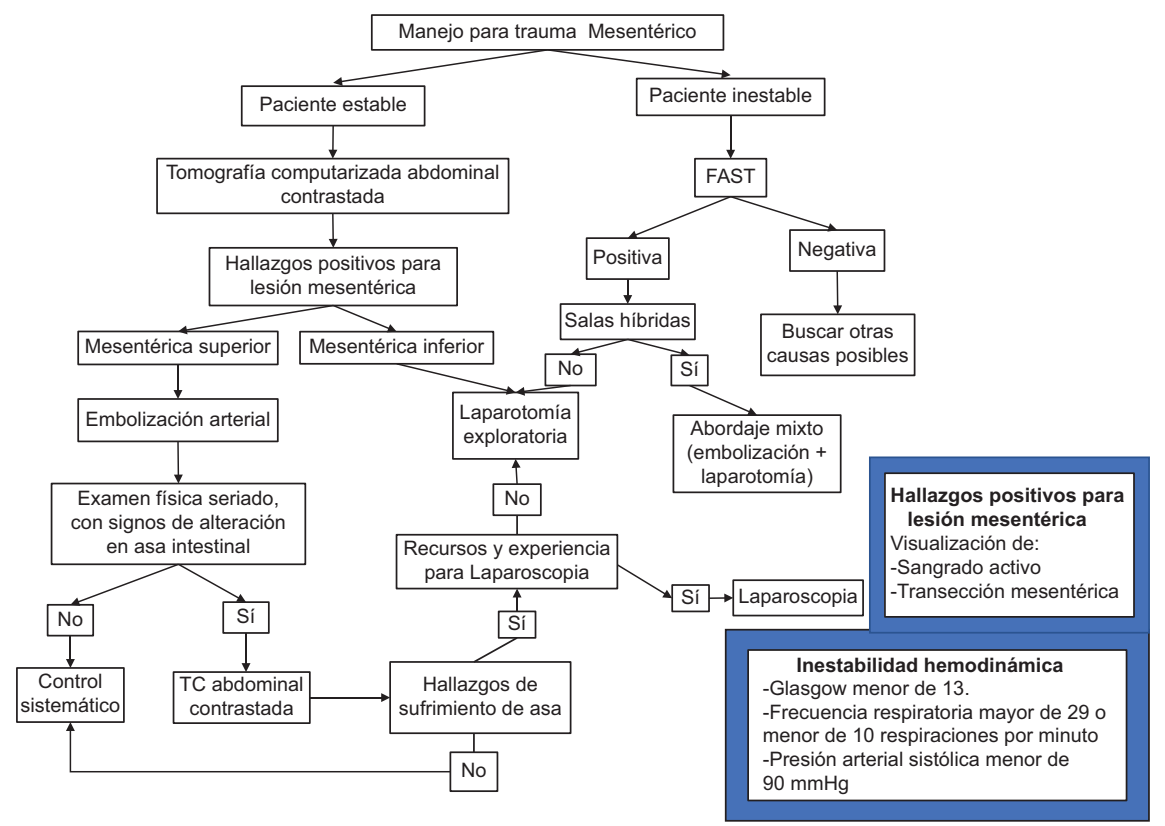

Figura 3. Algoritmo de manejo para trauma mesentérico, según nuestra experiencia y la revisión de la literatura. FAST: Focused Abdominal Sonography for Trauma; TC: tomografía computarizada.

disminuye el shock hemorrágico por pérdida de sangre antes de llegar al quirófano ${ }^{13}$. De otra manera, los que sí responden o desde el principio están hemodinámicamente estables deben ser tratados según su lesión. Cuando la afectada es la arteria mesentérica superior, el abordaje principal es la embolización para controlar el sangrado, como en el caso expuesto, y esto se debe a que el acceso de este vaso por cirugía abierta es complicado, y cuando exista perforación intestinal se puede hacer una segunda intervención después de controlar el sangrado, por medio de laparotomía o laparoscopia, según la experiencia del cirujano y los recursos. En cambio, cuando la afectada es la mesentérica inferior, el abordaje de preferencia es la laparotomía, ya que es más difícil hacer la embolización de esta arteria ${ }^{1,2}$.

Después de la embolización mesentérica se debe realizar un control estricto de los pacientes, ya que anteriormente no se consideraba esta técnica porque este tipo de lesiones se asociaban a perforación intestinal. El seguimiento debe ser con exploraciones físicas seriadas y control imagenológico. Si se detectara alguna anormalidad, habría que considerar la exploración quirúrgica ${ }^{13}$.

En un estudio retrospectivo realizado en 2015 por Ghelfi, et al. ${ }^{2}$, entre 335 pacientes atendidos entre los años 2007 y 2014 por traumatismo abdominal cerrado siete tuvieron lesión mesentérica sin signos de isquemia y con estabilidad hemodinámica, y fueron llevados a embolización arterial. Se logró el éxito en seis de los intervenidos, ya que un caso tuvo que ser convertido a laparotomía de emergencia para control de la hemorragia. Dentro de las complicaciones posteriores al procedimiento inicial, se presentó isquemia intestinal en un paciente. Por otro lado, en un reporte de caso presentado en 2019 por Nakama, et al. ${ }^{13}$, en el cual se evaluó el tratamiento de embolización mesentérica en un paciente posterior a un accidente automovilístico, este presentó deterioro clínico durante la exploración física seriada y requirió una nueva evaluación imagenológica, que confirmó necrosis intestinal y por ello el paciente fue llevado a laparotomía. Por esto se da importancia a la exploración física seriado tras la embolización, como se hizo en nuestro caso.

El manejo de las lesiones mesentéricas no está bien establecido y muchos de los cirujanos que se enfrentan a estos casos no tienen la experiencia suficiente para reconocerlos por su aparición tan infrecuente ${ }^{11,14}$. Su presentación conlleva un alto riesgo de mortalidad porque los vasos mesentéricos tienen a su cargo un $20-25 \%$ del gasto cardiaco ${ }^{11}$. Por ello, hemos elaborado un algoritmo basado en la literatura y en nuestra experiencia para este tipo de alteraciones (Fig. 3). 


\section{Conclusiones}

La lesión mesentérica es un complicación rara que se presenta después de un traumatismo abdominal cerrado. El manejo va a depender del estado hemodinámico del paciente. En los que exista un deterioro hemodinámico, la mejor técnica es la laparotomía, y si hay estabilidad hemodinámica se puede hacer una evaluación más completa mediante tomografía computarizada para determinar la estructura vascular comprometida y decidir cuál es el mejor método entre embolización o cirugía abierta.

\section{Conflicto de intereses}

Los autores declaran no tener ningún conflicto de intereses respecto a la publicación de este documento.

\section{Agradecimientos}

Los autores agradecen a la Universidad El Bosque por inculcar la labor investigadora en sus alumnos.

\section{Responsabilidades éticas}

Protección de personas y animales. Los autores declaran que para esta investigación no se han realizado experimentos en seres humanos ni en animales.

Confidencialidad de los datos. Los autores declaran que han seguido los protocolos de su centro de trabajo sobre la publicación de datos de pacientes.

Derecho a la privacidad y consentimiento informado. Los autores han obtenido el consentimiento informado de los pacientes y/o sujetos referidos en el artículo. Este documento obra en poder de los autores.

\section{Bibliografía}

1. Frandon J, Arvieux C, Thony F. Indications for embolization in a French level 1 trauma center. J Visc Surg. 2016;153:25-31.

2. Ghelfi J, Frandon J, Vendrell A, Rodiere M, Sengel C, Bricault I, et al. Arterial embolization in the management of mesenteric bleeding secondary to blunt abdominal trauma. Cardiovasc Intervent Radiol. 2016;39:683-9.

3. Advanced trauma life support, ATLS $\otimes$. Curso avanzado para cirujanos, 10. ${ }^{a}$ ed. Chicago: American College of Surgeons; 2018.

4. Lannes F, Scemama U, Maignan A, Boyer L, Beyer-Berjot L, Berdah SV, et al. Value of early repeated abdominal CT in selective non-operative management for blunt bowel and mesenteric injury. Eur Radiol. 2019;29:5932-40

5. Filiberto DM, Afzal MO, Sharpe JP, Seger C, Shankar S, Croce MA, et al. Radiographic predictors of therapeutic operative intervention after blunt abdominal trauma: the RAPTOR score. Eur J Trauma Emerg Surg. 2020 Apr 16. doi: 10.1007/s00068-020-01371-8. Online ahead of print.

6. Faget C, Taourel P, Charbit J, Ruyer A, Alili C, Molinari N, et al. Value of CT to predict surgically important bowel and/or mesenteric injury in blunt trauma: performance of a preliminary scoring system. Eur Radiol. 2015;25:3620-8.

7. Cinquantini F, Tugnoli G, Piccinini A, Coniglio C, Mannone S, Biscardi A, et al. Educational review of predictive value and findings of computed tomography scan in diagnosing bowel and mesenteric injuries after blunt trauma: correlation with trauma surgery findings in 163 patients. Can Assoc Radiol J. 2016;68:276-85.

8. Bates DD, Wasserman M, Malek A, Gorantla V, Anderson SW, Soto JA, et al. Multidetector CT of surgically proven blunt bowel and mesenteric injury. Radiographics. 2017;37:613-25.

9. Firetto MC, Sala F, Petrini M, Lemos AA, Canini T, Magnone S, et al. Blunt bowel and mesenteric trauma: role of clinical signs along with CT findings in patients' management. Emerg Radiol. 2018;25:461-7.

10. Shin JS, Shin JH, Ko HK, Kim JW, Yoon HK. Transcatheter arterial embolization for traumatic mesenteric bleeding: a 15-year, single-center experience. Diagn Interv Radiol. 2016;22:385-9.

11. Phillips B, Reiter S, Murray EP, McDonald D, Turco L, Cornell DL, et al Trauma to the superior mesenteric artery and superior mesenteric vein: a narrative review of rare but lethal injuries. World J Surg. 2017;42:713-26.

12. Adusumilli S, Gosselink MP, Ctercteko G, Pathmanathan N, El-Khoury T, Dutton $P$, et al. The efficacy of selective arterial embolization in the management of colonic bleeding. Tech Coloproctol. 2013;18:529-33.

13. Nakama R, Izawa Y, Kujirai D, Yagami T, Kono I, Tanimura K, et al. Transcatheter arterial embolization for initial hemostasis in a hemodynamically unstable patient with mesenteric hemorrhage: a case report. Radiol Case Rep. 2018;14:251-4.

14. McNutt MK, Chinapuvvula NR, Beckmann NM, Camp EA, Pommerening MJ, Laney RW, et al. Early surgical intervention for blunt bowel injury: the Bowel Injury Prediction Score (BIPS). J Trauma Acute Care Surg. 2015;78:105-11. 